TITLE:

\title{
Experimental performance study of STBC-based cooperative and diversity relaying
}

$\operatorname{AUTHOR}(S)$ :

Miyagoshi, Makoto; Murata, Hidekazu

\section{CITATION:}

Miyagoshi, Makoto ...[et al]. Experimental performance study of STBC-based cooperative and diversity relaying. IEICE Transactions on Fundamentals of Electronics,

Communications and Computer Sciences 2020, E103.A(5): 798-801

ISSUE DATE:

2020-05-01

URL:

http://hdl.handle.net/2433/260579

\section{RIGHT:}

(C) 2020 The Institute of Electronics, Information and Communication Engineers; 許諾条件に基づいて掲載しています。 


\title{
LETTER \\ Experimental Performance Study of STBC-Based Cooperative and Diversity Relaying
}

\author{
Makoto MIYAGOSHI $^{\dagger *}$, Nonmember and Hidekazu MURATA ${ }^{\dagger a)}$, Senior Member
}

SUMMARY The packet error rate (PER) performance of multi-hop STBC based cooperative and diversity relaying systems are studied. These systems consist of a source, a destination, and two relay stations in each hop. From in-lab experiments, it is confirmed that the cooperative relaying system has better PER performance than the diversity relaying system with highly correlated channels.

key words: multi-hop transmission, space-time block code, packet error rate, cooperative relaying, transmission experiment

\section{Introduction}

The performance of wireless systems can be improved by the multi-hop transmission [1], where signals are relayed in multiple hops between the source and the destination. The main advantage of the multi-hop transmission is that it extends the coverage and mitigates the effect of shadowing. This feature is useful for social infrastructures in water environment fields [2] and monitoring applications for agriculture and disaster prevention. However, in a simple multi-hop system, the endto-end packet error rate (PER) degrades with the number of hops.

To improve the performance of the multi-hop wireless network, a cooperative relaying system is proposed [3]. In this system, relay stations in each hop employ a transmit diversity technique and forward at the same time and frequency. The typical transmit diversity technique is spacetime block code (STBC) [4]. Using error detection codes and forwarding only the packets decoded correctly, there are conditions where the end-to-end PER performance of this system improves with the number of hops [5]. As a result, the cooperative relaying system achieves quite superior PER performance to the simple multi-hop system.

In the cooperative relaying system, relay stations in each hop assumed to work independently. The PER performance can be further improved if the received data packet is shared between relay stations. A diversity relaying system, where the correct packet is shared between relay stations in each hop, can achieve better performance than that of the cooperative relaying system under the same conditions. In the diversity relaying system, however, distances between cooperative relay stations are short so that relay stations can share

\footnotetext{
Manuscript received October 3, 2019.

Manuscript revised January 11, 2020.

$\dagger$ The authors are with the Graduate School of Informatics, Kyoto University, Kyoto-shi, 606-8501 Japan.

* Presently, the author is with NTT DOCOMO INC.

a) E-mail: murata@i.kyoto-u.ac.jp

DOI: $10.1587 /$ transfun.2019EAL2138
}

their received correct packets. The diversity gain at the diversity relaying system may be degraded due to the correlated propagation environments. Therefore, whether the diversity relaying system achieves superior PER performance to the cooperative relaying system or not depends on propagation channels.

The implementation and experimental results of cooperative communications are reported in several papers [6][8]. However, to the best of the authors' knowledge, there has been no work evaluating the performance of cooperative and diversity relaying systems, with focus on the effect of correlations between antennas. The goal of this study is to evaluate the end-to-end PER performance of multi-hop cooperative and diversity relaying systems in actual propagation environments. In this letter, in-lab experiments using transceivers and a fading emulator are performed to measure the end-to-end PER performance of STBC-based multi-hop cooperative and diversity relaying systems with correlated Rayleigh fading channels [9], while outdoor experiments are left for future work.

\section{System Model}

We consider STBC-based cooperative and diversity relaying systems having two relay stations in each hop as shown in Fig. 1. In these systems, each relay station performs error detection on their received packets and forwards only the

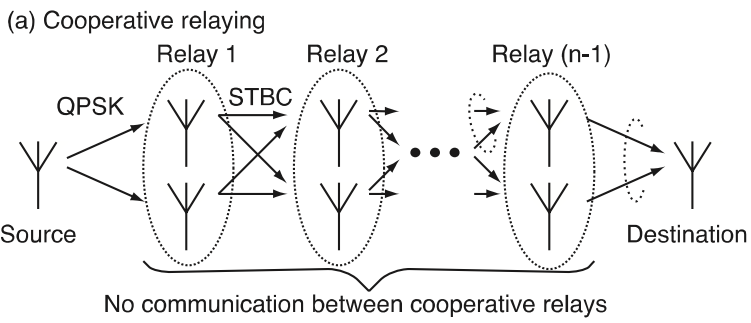

(b) Diversity relaying

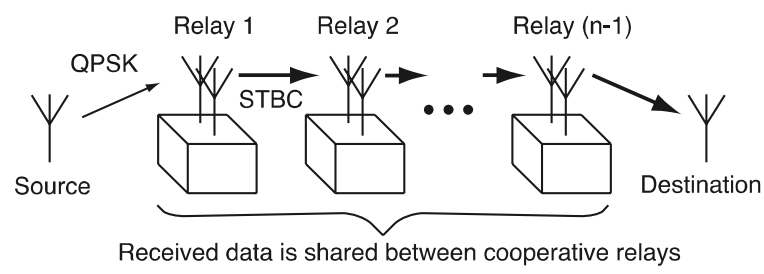

Fig. 1 System models: (a) STBC-based cooperative relaying systems (b) STBC-based diversity relaying systems. 
packets decoded correctly. In the diversity relaying system, the correct packets are shared between two relay stations, whereas in the cooperative relaying system, two relay stations work independently. Error detection is based on cyclic redundancy check (CRC). Two relay stations in each hop always perform STBC transmission. In this letter, the performance is measured in terms of the PER. In actual relaying systems, a routing process is necessary for assignments of orthogonal codes etc., however, the routing algorithm is out of the scope of this letter.

\section{Theoretical Packet Error Rate}

In this section, we describe the theoretical end-to-end PER of the cooperative relaying system [5], [10] and the diversity relaying system. Propagation channels are assumed as i.i.d. Rayleigh fading channels. The transmission power of the relay stations and the distance of each hop are assumed to be the same. These assumptions help us understand the behavior of the multi-hop cooperative and diversity relaying systems, and simplify the theoretical analysis.

\subsection{Cooperative Relaying}

In this system, STBC is employed in order to achieve the diversity gain. The single-hop PER therefore varies with the number of transmitting stations. There are three states in each hop and we define $S_{2}, S_{1}$ and $S_{0}$ as states, where two, one, and no relay station succeed in receiving and forward the received packets.

We also denote the probability distribution at $k$-th hop as $\boldsymbol{w}_{k}=\left[w_{2}^{(k)}, w_{1}^{(k)}, w_{0}^{(k)}\right]^{\mathrm{T}}$, where $w_{2}^{(k)}, w_{1}^{(k)}$, and $w_{0}^{(k)}$ show the ratio of $S_{2}, S_{1}$, and $S_{0}$ at $k$-th hop, and [. $]^{\mathrm{T}}$ denotes the transposed vector. The probability distribution at $(k+1)$-th hop can be shown as

$$
\begin{aligned}
\boldsymbol{w}_{k+1} & =\left[\begin{array}{ccc}
\left(1-p_{2}\right)^{2} & \left(1-p_{1}\right)^{2} & 0 \\
2 p_{2}\left(1-p_{2}\right) & 2 p_{1}\left(1-p_{1}\right) & 0 \\
p_{2}^{2} & p_{1}^{2} & 1
\end{array}\right] \boldsymbol{w}_{k} \\
& =\boldsymbol{P} \boldsymbol{w}_{k},
\end{aligned}
$$

where $p_{2}$ and $p_{1}$ show the single-hop PER with two cooperative transmitters and one transmitter, and $\boldsymbol{P}$ shows the transition matrix.

One source station transmits packets at the first hop; therefore, we get $\boldsymbol{w}_{0}=[0,1,0]^{\mathrm{T}}$. In addition, at the final hop, one destination station receives packets and the PER is shown by $p_{2}, p_{1}$ and 1 . Finally, we get the end-to-end PER of $n$-hop cooperative relaying system as

$$
\left[\begin{array}{lll}
p_{2} & p_{1} & 1
\end{array}\right] \boldsymbol{P}^{n-1} \boldsymbol{w}_{0}
$$

\subsection{Diversity Relaying}

In the diversity relaying system, two relay stations can forward the correct packet if one out of two relay station succeed

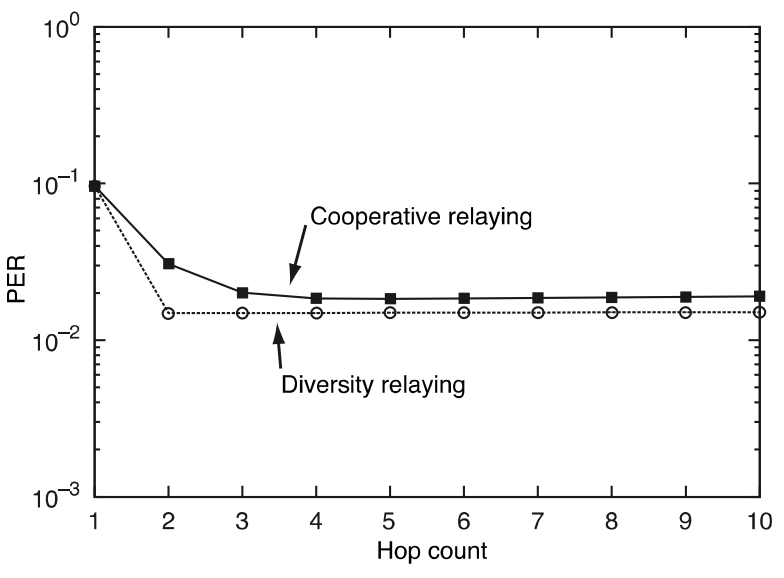

Fig. 2 Theoretical end-to-end PER performance.

to receive the packet correctly. The probability distribution at $(k+1)$-th hop can be written as

$$
\boldsymbol{w}_{k+1}^{\prime}=\left[\begin{array}{ccc}
1-p_{2}^{2} & 1-p_{1}^{2} & 0 \\
0 & 0 & 0 \\
p_{2}^{2} & p_{1}^{2} & 1
\end{array}\right] \boldsymbol{w}_{k}^{\prime}=\boldsymbol{P}^{\prime} \boldsymbol{w}_{k}^{\prime} .
$$

Similar to cooperative relaying, we get the end-to-end PER of $n$-hop diversity relaying system as

$$
\left[\begin{array}{lll}
p_{2} & p_{1} & 1
\end{array}\right] \boldsymbol{P}^{\prime n-1} \boldsymbol{w}_{0}
$$

\subsection{Numerical Examples}

For example, $p_{1}=9.65 \times 10^{-2}$ and $p_{2}=5.59 \times 10^{-3}$, these values are obtained from a former in-lab experiment [10]. The average SNR for each link is $19 \mathrm{~dB}$. The theoretical endto-end PER can be calculated from (1) and (2) and is shown in Fig. 2. We can see from Fig. 2 that, the diversity relaying system has slightly superior end-to-end PER performance to the cooperative relaying system with the same propagation channel.

\section{In-Lab Experiments}

In this section, we evaluate the PER performance of cooperative and diversity relaying systems using transceivers and a fading emulator. The block diagram and the overview of experimental setup are shown in Fig. 3. This system consists of universal software radio peripheral (USRP) N210s as transceivers, personal computers (PCs) as processors for digital baseband signals from USRPs, global positioning system (GPS) receivers for synchronizing USRPs, and the fading emulator as propagation channels. This system is a 3-hop relaying system. In the diversity relaying system, the packets received correctly are shared in two relay stations except training symbols.

Accuracy of timing and frequency synchronization have quite a big impact on the PER performance of the systems using STBC. In this system, one-pulse-per-second (1 PPS) signals and reference signals from onboard GPS disciplined 


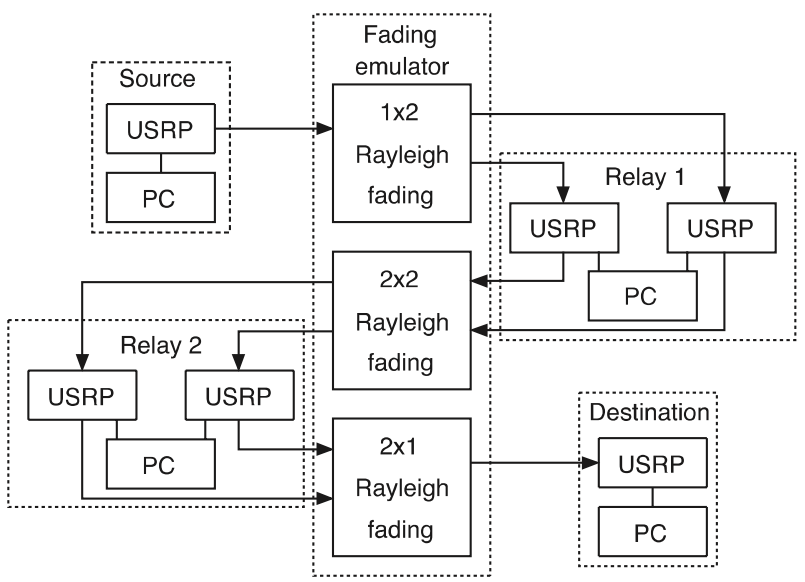

Fig. 3 Block diagram of experimental setup.

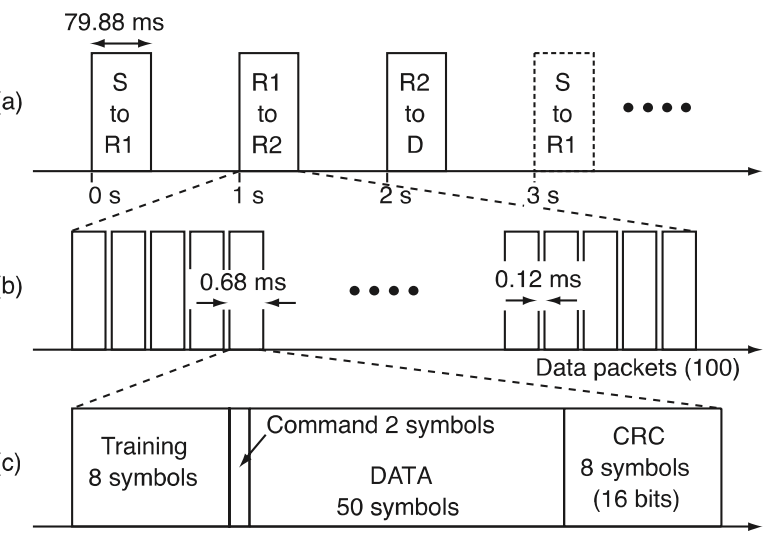

Fig. 4 Signal format.

oscillators (GPSDOs) are employed for timing and frequency synchronization.

\subsection{Transmission Format}

The structure of a transmit signal is shown in Fig. 4. In our system, PCs are not running a real-time operating system; therefore, the time required for signal processing is not constant. 100 packets are packed into one block so that communication overheads between USRPs and PCs are reduced. The blocks are transmitted at sufficiently long intervals in order to ensure proper signal processing on PCs.

The structure of packets is shown in Fig. 4(c). The packet consists of the training sequence, command symbols, data symbols, and CRC symbols. Two orthogonal training sequences are assigned uniquely for two relay stations in each hop. The modulation scheme is QPSK except for the training sequence. Command bits are utilized to control other stations from the source station. Data bits are the pseudo-random sequences. Command bits and data bits are fed to the CRC-16 encoder.

For pulse shaping, eight times oversampling and a root roll-off Nyquist filter with a roll-off factor of 0.7 are employed at the transmitter side. At the receiver side, over-
Table 1 Measurement system parameters.

\begin{tabular}{l|r}
\hline Parameters & Values \\
\hline Number of hops & 3 \\
Radio frequency & $5.109375 \mathrm{GHz}$ \\
Modulation scheme & QPSK \\
Symbol rate & 100 symbols/s \\
Oversampling & 8 samples/symbol \\
Packet length & 68 symbols \\
Filter & Root roll-off Nyquist \\
& (Roll-off factor $\alpha=0.7$ ) \\
STBC & Alamouti scheme \\
Error detection & CRC-16 \\
Channel model & Rayleigh fading \\
Average received SNR & $15 \mathrm{~dB}$ \\
Doppler frequency & $5 \mathrm{~Hz}$ \\
Antenna correlation coefficient & \\
$\quad$ in cooperative relaying & 0.0 \\
in diversity relaying & $0.0,0.6,0.9$ \\
\hline
\end{tabular}

sampled signals are utilized only for finding the symbol timing. The symbol timing is estimated by a simple correlation technique using the training sequence, and the channel state information is also acquired. Note that these processes are performed for each packet independently.

\subsection{System Parameters}

Parameters of the system are summarized in Table 1. We set the long-term average received signal to noise ratio (SNR) for each link as $15 \mathrm{~dB}$. Both transmit and receive antenna correlation coefficients (ACC) are set to 0.0 for cooperative relaying and $0.0,0.6,0.9$ for diversity relaying.

\section{Experimental Results}

The PER performance of cooperative and diversity relaying systems are presented in Fig. 5. PER at 1st and 2nd hop are the average values of two PER measured at two relay stations in each hop. ACCs are set to 0.0 for the cooperative relaying system because we assume the distance between two relays can be long enough. We can see from Fig. 5 that the diversity relaying system with ACCs 0.0 has superior PER performance to the cooperative relaying system. We also see from Fig. 5 that the PER performance of the diversity relaying system degrades with the increase of ACC. The diversity relaying system with ACC 0.6 and the cooperative relaying system achieve almost the same PER performance at 3rd hop. The diversity relaying system with ACC 0.9 is inferior in the PER performance to the cooperative relaying system.

The calculated PERs at the 2nd and the 3rd hops are also shown in Fig. 5. These PERs are obtained using the measured $p_{1}$ and $p_{2}$, and approximately agree with the experimental PERs. Since the measured PERs fluctuate trial by trial, we can observe small differences between the experimental PERs and the calculated PERs. Probabilities of $S_{2}$, $S_{1}$, and $S_{0}$, where two, one, and no relay station succeeded in receiving the packet correctly in these experiments, are shown in Table 2. In cooperative and diversity relaying systems, the PER performance improves with the number of 


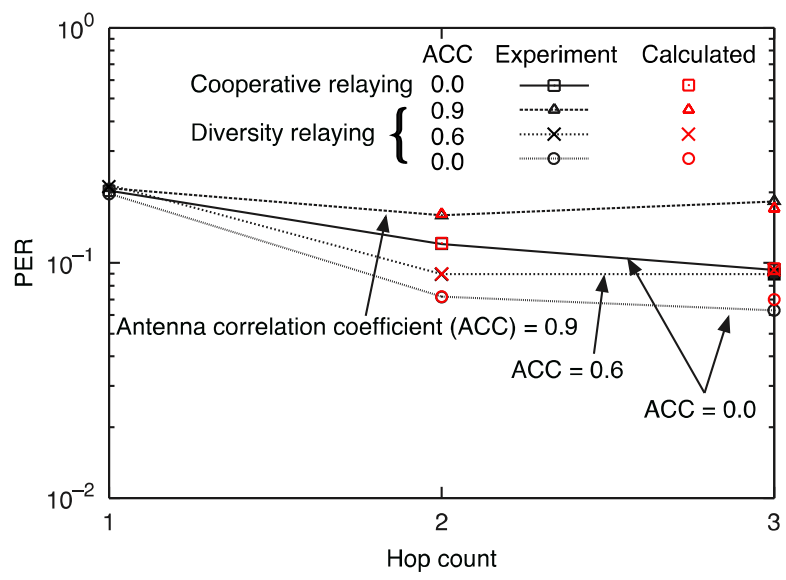

Fig. 5 PER performance of cooperative and diversity relaying.

Table 2 Percentage of $S_{2}, S_{1}$, and $S_{0}$, where two, one, and no relay station succeeded in receiving the packet correctly.

\begin{tabular}{ll|rr}
\hline \multirow{2}{*}{$\begin{array}{l}\text { Antenna correlation } \\
\text { coefficient (and mode) }\end{array}$} & State & \multicolumn{2}{|c}{ Position } \\
\cline { 2 - 4 } & & Relay1 & Relay2 \\
\hline \multirow{3}{*}{0.0 (cooperative) } & $S_{2}$ & $63.0 \%$ & $86.9 \%$ \\
\cline { 2 - 4 } & $S_{1}$ & $33.4 \%$ & $8.2 \%$ \\
\cline { 2 - 4 } & $S_{0}$ & $3.6 \%$ & $4.9 \%$ \\
\hline \multirow{3}{*}{0.0 (diversity) } & $S_{2}$ & $64.0 \%$ & $89.2 \%$ \\
\hline & $S_{1}$ & $32.5 \%$ & $7.2 \%$ \\
\hline \multirow{3}{*}{0.6 (diversity) } & $S_{0}$ & $3.5 \%$ & $3.6 \%$ \\
\hline \multirow{3}{*}{0.9 (diversity) } & $S_{2}$ & $61.6 \%$ & $86.9 \%$ \\
\cline { 2 - 4 } & $S_{1}$ & $33.7 \%$ & $8.2 \%$ \\
\cline { 2 - 4 } & $S_{0}$ & $4.6 \%$ & $4.9 \%$ \\
\hline & $S_{2}$ & $67.7 \%$ & $80.0 \%$ \\
\hline & $S_{1}$ & $23.0 \%$ & $8.2 \%$ \\
\hline & $S_{0}$ & $9.3 \%$ & $11.8 \%$ \\
\hline
\end{tabular}

hops because probability of $S_{1}$ reduces and the probability of $S_{2}$ increases from the initial state distribution [5], [10]. The probability of $S_{0}$ play an important role on the lower limit of the PER performance. From Table 2, we see the probability of $S_{0}$ increases with ACC, that is, the diversity gain is degrades with ACC. From Table 2 and Fig. 5, the cooperative relaying system can achieve better PER performance than the diversity relaying system with highly correlated channel states due to the degradation on the diversity gain of the diversity relaying system.

\section{Conclusion}

In this letter, the PER performance of the STBC based co- operative and diversity relaying system is presented from in-lab experiments. It is confirmed from the experimental results that, the cooperative relaying system has better PER performance than the diversity relaying system with highly correlated channels. In future work, we will perform outdoor experiments using these implementations.

\section{Acknowledgments}

The present study was supported by the Strategic Information and Communications R\&D Promotion Programme (SCOPE) of the Ministry of Internal Affairs and Communications, Japan.

\section{References}

[1] Y.D. Lin and Y.C. Hsu, "Multihop cellular: A new architecture for wireless communications," Proc. IEEE INFOCOM'00, vol.3, pp.1273-1282, March 2000.

[2] Y. Sueyoshi, H. Morita, and K. Jin, "IoT solution system of water environment infrastructure facilities - Remote monitoring of facilities using wireless network -," IEICE Technical Report, RCS2018-90, July 2018.

[3] J.N. Laneman, D.N.C. Tse, and G.W. Wornell, "Cooperative diversity in wireless networks: Efficient protocols and outage behavior," IEEE Trans. Inf. Theory, vol.50, no.12, pp.3062-3080, Dec. 2004.

[4] V. Tarokh, H. Jafarkhani, and A.R. Calderbank, "Space-time block codes from orthogonal designs," IEEE Trans. Inf. Theory, vol.45, no.5, pp.1456-1467, July 1999.

[5] Y. Oishi, H. Murata, K. Yamamoto, and S. Yoshida, "Theoretical FER performance of multi-hop wireless cooperative networks using transmit diversity," Proc. IEEE VTC 2008-Spring, pp.2366-2369, May 2008.

[6] P. Murphy and A. Sabharwal, "Design, implementation, and characterization of a cooperative communication system," IEEE Trans. Inf. Theory, vol.60, no.6, pp.2534-2544, July 2011.

[7] T. Korakis, M. Know, E. Erkip, and S. Panwar, "Cooperative network implementation using open-source platforms," IEEE Commun. Mag., vol.47, no.2, pp.134-141, Feb. 2009.

[8] T. Mimura, A. Kuwabara, H. Murata, K. Yamamoto, and S. Yoshida, "Packet transmission experiments of STBC-based multi-hop cooperative relaying," Proc. IEEE ICC'11, pp.1-5, June 2011.

[9] M. Miyagoshi, H. Murata, S. Yoshida, K. Yamamoto, D. Umehara, S. Denno, and M. Morikura, "Experimental performance comparison of STBC-based cooperative and diversity relaying," Proc. IEEE VTS Asia Pacific Wireless Communications Symposium, Kyoto, Aug. 2012

[10] H. Murata, M. Miyagoshi, and Y. Oishi, "Analytical end-to-end PER performance of multi-hop cooperative relaying and its experimental verification," IEICE Trans. Commun., vol.E100-B, no.3, pp.449455, March 2017. 